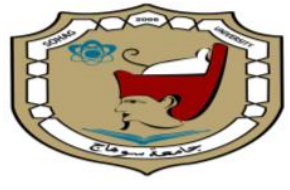

Sohag University

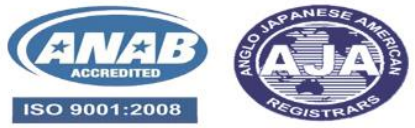

Sohag Medical Journal

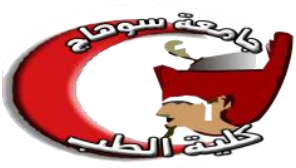

Faculty of Medicine

\title{
Non-motor features of essential tremor, Updated review of evolving aspects
}

\section{Gharib Fawi, Hazem K Elhewag, Mohamed Nasreldin Thabit, Ahmed Ezzat Amin}

Department of Neurology and Psychological medicine, Faculty of Medicine, Sohag University

\begin{abstract}
Essential tremor (ET) is defined as an action tremor syndrome with a duration of more than three years in both upper limbs with or without tremor in other areas and with no other neurological signs. ET's non-motor characteristics add to the evidence of clinical variability in ET, a disease with a wide range of pathological and etiological characteristics. Problems in frontal-executive function, comparable to Parkinson's disease (PD), were one of the most common patterns of cognitive affection in ET. ET individuals who started tremor after the age of 65 were 64 to $70 \%$ more likely than control subjects to develop dementia, but ET cases who started tremor before the age of 65 have an equal chance to develop dementia as controls. Depression rates were considerably higher in ET than controls and more severe depression is found. A strong evidence linking anxiety and ET. Many studies have documented sleep disturbances in ET.

Keywords: essential tremor, non-motor features, cognition, depression, anxiety, sleep disturbances.
\end{abstract}

\section{Introduction:}

Essential tremor (ET) is defined as an action tremor syndrome with a duration of more than three years in both upper limbs with or without tremor in other areas like head, voice, lower limbs, and/or trunk and with no other neurological signs like dystonia, ataxia, and/or parkinsonism) ${ }^{[1]}$.

Rest tremors may develop in advanced $\mathrm{E}$ $\mathrm{T}$, but they do not subside following pur poseful movements which differentiates ET from parkinsonian tremor ${ }^{[2] . ~ T h e ~}$ non-motor features of ET contribute to the evidence of clinical heterogeneity in ET, an illness with heterogeneity in pathological and etiological features ${ }^{[3]}$.

\section{Cognition:}

ET has been linked to a wide range of levels of cognitive functions, from normal cognition to dementia, according to numerous research ${ }^{[4-6]}$. There is growing evidence that ET patients progress to mild cognitive impairment (MCI) and dementia at a faster rate than age-matched controls despite that the majority of ET patients perform within the normal cognitive spectrum ${ }^{[5,7]}$.

Gasparini et al. were among the first to discover that ET patients had severe frontal function impairments when compared to healthy controls ${ }^{[8]}$. In a recent case-control observational study on ET 
cases and matched healthy controls from Egypt were examined, and it was shown that patients with ET have significant cognitive dysfunction ${ }^{[9]}$. One of the most prominent patterns of cognitive affecttion in ET was problems with frontalexecutive function, which are similar to Parkinson's disease (PD) ${ }^{[8]}$ as well as those involving visual attention [10], verbal fluency, naming, mental set shifting, verbal memory, working memory [11], complex auditory attention, visual attention and response inhibition, recall of a word list, verbal fluency, visual confrontation naming, verbal fluency, immediate word list recall, semantic encoding, and facial matching ${ }^{[12]}$.

ET individuals who started tremor after the age of 65 were 64 to $70 \%$ more likely than control subjects to develop dementia, but ET cases who started tremor before the age of 65 have an equal chance to develop dementia as controls ${ }^{[5]}$. In the same context, an older-onset ET also was connected to a slight but statistically significant increase in the risk of MCI in a recent study, whereas youngeronset ET was not ${ }^{[4] \text {. }}$

The basis for cognitive dysfunction in ET is unclear. The role of the cerebellum in cognition is more and more becoming

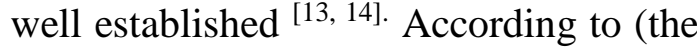
dysmetria of mind) idea, the cerebellum regulates the pace, consistency, capacity, and appropriateness of mental or cognitive processes, much as it regulates the rate, rhythm, force, and precision of motions $^{[14]}$, this could give an explanation for the cerebellum's role in cognitive processes. In a study by Stoodley et al., the hypothesis was tested by the fact that lesions in the posterolateral cerebellum cause the Cerebellar cognitive affective syndrome (CCAS; Schmahmann's syndrome) using voxel-based lesion-symptom mapping [15]. They found that patients with CCAS had damage to posterior lobe regions, with lesions resulting in affected scores on tests for language, spatial, and executive function even if they did not have cerebellar motor syndrome ${ }^{[15]}$. All of the abovementioned data suggest the role of cerebellum which is the main site of pathological changes in ET in producing cognitive dysfunction ${ }^{\text {[16-18]. }}$

Another explanation for ET's MCI and dementia came from postmortem examinations, which revealed that ET's brains had more Alzheimer's type alterations than age-matched control brains ${ }^{[19]}$. Alzheimer's disease affects the majority of ET patients who develop dementia, and the risk of Alzheimer's disease is substantially higher in ET patients, according to epidemiological studies ${ }^{[5,20]}$.

\section{Depression:}

Lombardi et al. were among the first to study depression in ET using the Geriatric Depression Scale to assess depresssion in 18 individuals with ET. They discovered that individuals had severe depression that did not correlate to the degree of their tremors [11]. Depression scores were considerably higher in ET than controls in an Indian research of 50 ET cases and 50 controls ${ }^{[21]}$. A study employing Beck Depression Inventory scores yielded the same outcome ${ }^{[6]}$. A significant prevalence of depression (more than half of cases) was discovered in a recent study in China, in particular women, cases with head or voice tremors, and those having significant functional impairment ${ }^{[22]}$.

Higher depression levels were linked to more severe tremors ${ }^{[23,21]}$. Some studies have discovered a link between depresssion and the total Fahn-Tolosa-Marn Tremor Rating Scale, which includes overall tremor as well as a self-reported functional impairment score, implying 
that depressive symptoms could be a secondary response to tremor not a primary feature of the disease ${ }^{[24,25]}$. A point of much debate about this is that others found no relation between depression and tremor severity ${ }^{[26] \text {. }}$

\section{Anxiety:}

A strong evidence linking anxiety and ET. In an Egyptian community research involving $30 \mathrm{ET}$ patients, who were shown to be more anxious than controls using Hamilton anxiety rating scale ${ }^{[9]}$. In a research involving 40 ET patients, using unilateral thalamic DBS for ET treatment, patients showed lower anxiety levels (measured by mood state) 3 and 12 months following surgery [27]. The relation between anxiety and ET severity is a matter of debate. Some studies found a correlation [28, 29], and others showed no correlation ${ }^{[30]}$.

\section{Sleep disturbances:}

Many investigations have established the presence of sleep problems in ET patients in the last few years [6, 24, 31-35]. Because of the probable importance of the locus calculus in sleep regulation, Lewy body depositions in this area are higher in ET patients' postmortem brains than in control brains, which could be linked to the development of sleep problems in this disease ${ }^{[36]}$.

This includes excessive daytime sleepiness ${ }^{[37,38-40]}$, rapid eye movements behavior disorder [41,42], restless leg syndrome $[43,44]$.

\section{Conclusion}

Non-motor features of ET have underestimated aspects of the disease which adds a significant burden on the patient besides the motor features. The cognitive issues in ET involve rapid progression $\mathrm{f}$ rom MCI to dementia and frontal lobe dysfunction. More prevalent depression and anxiety in ET cases is a well-established finding in many studies. Sleep disturbances in ET may be explained by the pathological changes in found the brain stem of ET cases. We recommend further studies on these and other nonmotor features in ET.

\section{References}

1. Bhatia, K.P., et al., Consensus Statement on the classification of tremors. from the task force on tremors of the International Parkinson and Movement Disorder Society. Movement Disorders, 2018. 33(1): p. 75-87.

2. Papengut, F., et al., Rest tremor suppression may separate essential from parkinsonian rest tremor. Parkinsonism $\&$ related disorders, 2013. 19(7): p. 693697.

3. Louis, E.D., S.J. Frucht, and E. Rios, Intention tremor in essential tremor: prevalence and association with disease duration. Movement disorders: official journal of the Movement Disorder Society, 2009. 24(4): p. 626.

4. Benito-Leon, J., et al., Elderly-onset essential tremor and mild cognitive impairment: a population-based study (NEDICES). J Alzheimers Dis, 2011. 23(4): p. 727-35.

5. Benito-Leon, J., et al., Elderly-onset essential tremor is associated with dementia. Neurology, 2006. 66(10): p. 1500-5.

6. Sengul, Y., et al., Cognitive functions, fatigue, depression, anxiety, and sleep disturbances: assessment of nonmotor features in young patients with essential tremor. Acta Neurologica Belgica, 2015. 115(3): p. 281-287.

7. Bermejo-Pareja, F., E.D. Louis, and J. Benito-León, Risk of incident dementia in essential tremor: a population-based study. Movement disorders: official 
journal of the Movement Disorder Society, 2007. 22(11): p. 1573-1580.

8. Gasparini, M., et al., Frontal lobe dysfunction in essential tremor: a preliminary study. J Neurol, 2001. 248(5): p. 399-402.

9. Elbelkemy, M., et al., Non motor symptoms in patients with essential tremor. QJM: An International Journal of Medicine, 2020. 113(Supplement_1).

10.Vermilion, K., A. Stone, and D. Duane, Cognition and affect in idiopathic essential tremor. Mov Disord, 2001. 16(suppl 1): p. S30.

11.Lombardi, W.J., et al., Cognitive deficits in patients with essential tremor. Neurology, 2001. 57(5): p. 785-90.

12.Tröster, A., et al., Neuropsychological deficits in essential tremor: an expression of cerebello-thalamo-cortical pathophysiology? European Journal of Neurology, 2002. 9(2): p. 143-151.

13.Rapoport, M., R. van Reekum, and $H$. Mayberg, The role of the cerebellum in cognition and behavior: a selective review. The Journal of neuropsychiatry and clinical neurosciences, 2000. 12(2): p. 193-198.

14. Schmahmann, J.D., The cerebellum and cognition. Neuroscience letters, 2019. 688: p. 62-75.

15.Stoodley, C.J., et al., Location of lesion determines motor vs. cognitive consequences in patients with cerebellar stroke. Neuroimage Clin, 2016. 12: p. 765-775.

16.Babij, R., et al., Purkinje cell axonal anatomy: quantifying morphometric changes in essential tremor versus control brains. Brain, 2013. 136(10): p. 3051-3061.

17.Delay, C., et al., Increased LINGO1 in the cerebellum of essential tremor patients. Movement Disorders, 2014. 29(13): p. 1637-1647.

18. Béliveau, E., et al., Accumulation of amyloid- $\beta$ in the cerebellar cortex of essential tremor patients. Neurobiology of disease, 2015. 82: p. 397-408.

19.Louis, E.D., et al., Neuropathological changes in essential tremor: 33 cases compared with 21 controls. Brain, 2007. 130(Pt 12): p. 3297-307.

20.Louis, E.D., et al., Faster rate of cognitive decline in essential tremor cases than controls: a prospective study. European journal of neurology, 2010. 17(10): p. 1291-1297.

21.Chandran, V., et al., Non-motor features in essential tremor. Acta neurologica scandinavica, 2012. 125(5): p. 332-337.

22.Huang, H., et al., Prevalence and Risk Factors of Depression and Anxiety in Essential Tremor Patients: A CrossSectional Study in Southwest China. Frontiers in Neurology, 2019. 10: p. 1194.

23.Miller, K.M., et al., Depression symptoms in movement disorders: comparing Parkinson's disease, dystonia, and essential tremor. Mov Disord, 2007. 22(5): p. 666-72.

24.Li, Z.-W., et al., Characteristics of depressive symptoms in essential tremor. Journal of Clinical Neuroscience, 2011. 18(1): p. 52-56.

25.Mao, C.J., et al., [Depression associated with movement disorders]. Zhonghua $\mathrm{Yi}$ Xue Za Zhi, 2013. 93(1): p. 26-9.

26.Lacritz, L.H., et al., Cognitive functioning in individuals with "benign" essential tremor. J Int Neuropsychol Soc, 2002. 8(1): p. 125-9.

27.Fields, J.A., et al., Neuropsychological and quality of life outcomes 12 months after unilateral thalamic stimulation for 
essential tremor. J Neurol Neurosurg

Psychiatry, 2003. 74(3): p. 305-11.

28. Topçuoğlu, V., et al., [Social phobia in essential tremor]. Turk Psikiyatri Derg, 2006. 17(2): p. 93-100.

29.Louis, E.D., et al., Risk of tremor and impairment from tremor in relatives of patients with essential tremor: a community-based family study. Annals of Neurology: Official Journal of the American Neurological Association and the Child Neurology Society, 2001. 49(6): p. 761-769.

30.Lundervold, D.A., P.A. Ament, and P. Holt, Social anxiety, tremor severity, and tremor disability: a search for clinically relevant measures. Psychiatry J, 2013. 2013: p. 257459

31.Louis, E.D., et al., Essential tremorParkinson's disease: A double whammy. J Neurol Sci, 2016. 366: p. 47-51.

32. Acar, B.A. and T. Acar, Essential Tremor is not Only a Movement Disorder; Its Relationship with Sleep and Anxiety. Noro Psikiyatr Ars, 2019. 56(1): p. 18 22.

33. Shalash, A.S., et al., Clinical profile of non-motor symptoms in patients with essential tremor: impact on quality of life and age-related differences. Tremor and Other Hyperkinetic Movements, 2019. 9.

34.Lee, S.M., et al., Nonmotor symptoms in essential tremor: Comparison with Parkinson's disease and normal control. J Neurol Sci, 2015. 349(1-2): p. 168-73.

35.Chen, J., et al., [Sleep disorders associated with essential tremor and Parkinson's disease]. Zhonghua Yi Xue Za Zhi, 2015. 95(3): p. 205-9.

36.Louis, E.D., Non-motor symptoms in essential tremor: A review of the current data and state of the field. Parkinsonism Relat Disord, 2016. 22 Suppl 1(0 1): p. S115-8.

37.Rohl, B., et al., Daytime sleepiness and nighttime sleep quality across the full spectrum of cognitive presentations in essential tremor. J Neurol Sci, 2016. 371: p. 24-31.

38.Jiménez-Jiménez, F.J., et al., Sleep Disorders in Essential Tremor: Systematic Review and Meta-Analysis. Sleep, 2020.

39.Gerbin, M., A.S. Viner, and E.D. Louis, Sleep in essential tremor: a comparison with normal controls and Parkinson's disease patients. Parkinsonism Relat Disord, 2012. 18(3): p. 279-84.

40.Adler, C.H., et al., Probable RBD is increased in Parkinson's disease but not in essential tremor or restless legs syndrome. Parkinsonism Relat Disord, 2011. 17(6): p. 456-8.

41.Lacerte, A., et al., Increased prevalence of non-motor symptoms in essential tremor. Tremor and other hyperkinetic movements, 2014. 4.

42. Salsone, M., et al., REM-Sleep Behavior Disorder in Patients With Essential Tremor: What Is Its Clinical Significance? Frontiers in Neurology, 2019. 10(315).

43.Alonso-Navarro, H., et al., Association between restless legs syndrome and other movement disorders. Neurology, 2019. 92(20): p. 948-964.

44.Wu, Y., et al., Prevalence and clinical features of non-motor symptoms of essential tremor in Shanghai rural area. Parkinsonism Relat Disord, 2016. 22: p. 15-20. 